\title{
Double diffusion natural convection in a square cavity filled with nanofluid
}

\author{
Sheng Chen ${ }^{1,2 *}$, Bo Yang ${ }^{2}$, Kai H. Luo ${ }^{3}$, Xiaopeng Xiong ${ }^{2}$, \\ Chuguang Zheng ${ }^{2}$ \\ 1 Department of Mechanical Engineering, University College London, London \\ WC1E 7JE, United Kingdom \\ 2 State Key Laboratory of Coal Combustion, Huazhong University of Science and \\ Technology, Wuhan 430074, China \\ 3 Department of Mechanical Engineering, University College London, London \\ WC1E 7JE, United Kingdom
}

\begin{abstract}
Double diffusive natural convection of nanofluid is commonly found in renewable energy engineering. However, nowadays our understanding on its fundamental characteristics is still limited. Especially, three crucial questions on its fundament have not been answered yet: (1) its performance not only in laminar regimes but also beyond laminar regimes, (2) the influence of the ratio of buoyancy forces on heat and mass transfer, (3) the correlation among the dimonsionless quantities which describe the features of this kind of convection. The present work tries to reveal the characteristics of double diffusion natural convection of nanofluid over a wide range, from laminar regimes to turbulent regimes, with the aid of numerical experiments. It is observed that the behavior of nanofluid in the laminar regimes is different from that in the turbulent regimes. Some conclusions presented in previous literatue for laminar double diffusion of nanofluid may be invalid in its turbulent counterpart. The effect of the ratio of buoyancy forces on heat and mass transfer of nanofluid possesses some similarities with the pure base fluid as well as some obvious differences. Especially, a power-like correlation among the Nusselt number, Sherwood number, Rayleigh number, ratio of buoyancy forces and nanoparticl volume fraction has been extracted for the first time through our numerical experiments.
\end{abstract}

Key words: Nanofluid; turbulent natural convection; double diffusion; large eddy simulation; lattice Boltzmann method

$1 *$ Corresponding author.Faculty of Engineering, The University of Nottingham, University Park, Nottingham NG7 2RD, United Kingdom. 


\section{Introduction}

Nanofluid is a mixture of base fluid (e.g. water) and nanometer-sized particles. The suspending nanoparticles, typically made of metals, oxides, carbides, or carbon nanotubes, can significantly enhance the thermal conductivity of base fluid $[1,2]$. Consequently, as a promising new generation of coolant, nanofluid has a great potential adopted in many important applications such as microelectronics, domestic refrigerator and aircrafts. One can refer to Refs. [3-6] for the latest progress in this field.

Compared with the available numerous studies on the characteristics of heat transfer of nanofluid induced by thermal buoyancy [5], the exploration taking effects of compositional buoyancy into account is quite limited, although compositional buoyancy exists and plays an important role in many industrial applications utilizing nanofluid, such as solar energy industry[7]. Esfahani and Bordbar [8] perhaps are some of the pioneers on this topic. In Ref.[8], they investigated laminar double-diffusive natural convection heat transfer enhancement in a square enclosure filled with various nanofluids by numerical simulation. The influences of the nanoparticle volume fraction, Rayleigh and Lewis number on the Nusselt and Sherwood number were discussed. Later, Parvin et al. [9] numerically analyzed the flow and natural convection patterns of water- $\mathrm{Al}_{2} \mathrm{O}_{3}$ nanofluid in a partially heated enclosure. In their study, the nanoparticle volume fraction varies from 0 to 0.2 with the Rayleigh number up to $10^{6}$. Through their work, it is very clear that the distributions of isotherms and iso-concentrations depend closely on the position of active walls of the cavity. Recently, laminar double diffusion in a solar collector using water-CuO nanofluid was modelled in Ref. [10]. The cross section of the solar collector is triangular. The authors revealed the incident angle of the solar collector influenced the performance of heat and mass transfer of nanofluid significantly. The above publications all focus on the behavior of double diffusion of nanofluid confined by a closed container. The double diffusive convection in a nanofluid layer was firstly reported by Nield and Kuznetsov $[11,12]$. They discussed the onset and thickness of such layer through analytical study. In succession, the same authors extended their discussion to a porous medium saturated by nanofluid [13]. More recently, Beg and Tripathi [14] conducted a theoretical study on double diffusive convection in nanofluid through a deformable channel. Through the work, the authors tried to deepen our understanding on the usability of nanofluid in physiological areas.

Through the above literature survey it is clear that at least three fundamental questions on double diffusion of nanofluid are not answered yet: firstly, the characteristics of double diffusion of nanofluid beyond laminar regimes; secondly, the influence of the ratio of buoyancy forces on heat and mass transfer; and thirdly, the correlation among the Nusselt/Sherwood number, Rayleigh 


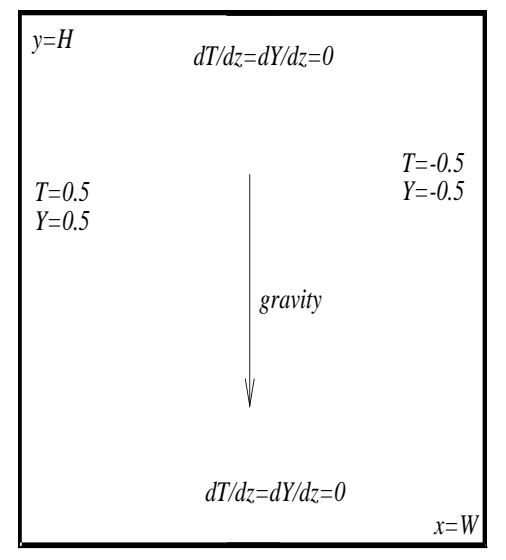

Fig. 1. Configuration of the computational domain and boundary conditions.

number and nanoparticle volume fraction under double diffusive natural convection. The goal of this paper is to answer these three critical questions with the aid of a comprehensive numerical experiment.

\section{Investigated domain and boundary conditions}

In this work, double diffusion in a square cavity $[8,15]$ is adopted as it is a good research prototype to reply the above questions. The investigated domain and boundary conditions are illustrated by Fig.1. The dimensionless length of each side of the square cavity is unity. The dimensionless temperature and concentration on the hot wall and cold wall read $T_{h}=0.5, Y_{h}=0.5$ and $T_{c}=0.5, Y_{c}=0.5$, respectively. The top and bottom walls of the investigated domain are adiabatic. The gravity $g$ is downward. The investigated domain and boundary conditions are identical to that in Ref.[15] where one can find the more detailed description on this investigated domain.

\section{Governing equations}

For nanofluid simulation, generally there are two ways: single phase and twophase modelling [16]. In the former approach it is assumed that the suspending nanoparticles are in thermal equilibrium with the base fluid and there is no velocity slip between the solid particles and base fluid. Therefore the solid-liquid mixture can be treated as a kind of Newtonian fluid. However, in the latter, the discrete phase and continuum phase are described in a Lagrangian and a Eulerian scheme, respectively. Although it is physical sound over broader ranges than single phase approach, two-phase modelling suffers from a higher 
requirement on computational resources. If the solid volume fraction is small enough, single phase simplification can provide acceptable predictions for the heat transfer and hydrodynamic properties of nanofluid with cheaper computational cost. Consequently, single phase approach is popularly utilized for simulating double-diffusive natural convection of nanofluid [8-10]. The present study is also based on this approach.

To date there have been three categories of numerical methods to investigate flows beyond laminar regimes: Reynolds averaged Navier-Stokes equations (RANS), large eddy simulation (LES) and direct numerical simulation (DNS). Among them, LES is much cheaper than DNS regarding computational cost and can capture more turbulence details than RANS. As a result, in the present work LES is employed to numerically analyze double diffusion of nanofluid.

With the aid of the normalizing process introduced in Ref.[15], the corresponding dimensionless governing equations in the LES framework read[8,15]

$$
\begin{aligned}
& \nabla \cdot \vec{u}=0 \\
& \frac{\partial \vec{u}}{\partial t}+\vec{u} \cdot \nabla \vec{u}=-\nabla p+\nu_{e} \Delta \vec{u}-\operatorname{Pr}(T-N Y) \frac{\vec{g}}{|\vec{g}|} \\
& \frac{\partial T}{\partial t}+\vec{u} \cdot \nabla T=\alpha_{e} \Delta T \\
& \frac{\partial Y}{\partial t}+\vec{u} \cdot \nabla Y=D_{e} \Delta Y
\end{aligned}
$$

where $\nu_{e}, \alpha_{e}$ and $D_{e}$ are the effective viscosity, thermal and solutal diffusivity, respectively. $p$ is the pressure, $\rho$ is the density $T$ is the temperature and $Y$ is the concentration. $\vec{u}=(u, v)$ is the velocity vector. The gravity $\vec{g}$ downward. $N$ is the ratio of buoyancy forces, $R a$ is the Rayleigh number and $\operatorname{Pr}$ is the Prandtl number.

In the single phase approach for nanofluid simulation, the influences of addition of nanoparticles into base fluid are reflected by the change of viscosity and thermal conductivity of fluid [16,17]. Nowadays there are a number of models to predict these fundamental physical properties of nanofluid [16] and it has been found that the numerical results depend closely on the adopted models [17]. Even for laminar flow, the trends of numerical predictions may be completely inverse as a result of different models of viscosity and thermal conductivity used in simulation [17]. Unfortunately, until now there is still no consensus on this issue [16]. To avoid the uncertainties, the physical properties of water-SiO $\mathrm{S}_{2}$ nanofluid obtained through experimental measure[18], rather than by the models [16], are adopted in this work. 


\section{Numerical method}

The lattice Boltzmann (LB) LES model, developed in our previous work [15], for turbulent double diffusion simulation is adopted in this study.

The evolution equation for the flow field reads

$$
g_{k}\left(\vec{x}+c \vec{e}_{k} \Delta t, t+\Delta t\right)-g_{k}(\vec{x}, t)=\Omega_{k}(g)+\Delta t F_{k}
$$

where $g_{k}(\vec{x}, t)$ is the distribution function associated with the fluid particle moving with the discrete velocity $\vec{e}_{k} . c=\Delta x / \Delta t$ is the fluid particle speed. $\Delta x$ and $\Delta t$ are the lattice grid spacing and the time step, respectively. The term $F_{k}$ is a forcing term accounting for the body force experienced by the fluid particle, and $\Omega_{k}(g)$ is the discrete collision operator. For multiple-relaxationtimes (MRT) models, the collision operator is given by

$$
\Omega_{k}(g)=-\sum_{j}\left(\mathbf{M}^{-1} \mathbf{S M}\right)_{j k}\left(g_{j}-g_{j}^{(e q)}\right)
$$

where $g_{j}^{(e q)}$ is the discrete equilibrium distribution function and $\mathbf{S}=\operatorname{diag}\left(\tau_{0}, \tau_{1}, \ldots, \tau_{k-1}\right)^{-1}$ is a non-negative diagonal matrix. For the D2Q9 model

$$
\vec{e}_{k}= \begin{cases}(0,0) & : \quad k=0 \\ (\cos (k-1) \pi / 2, \sin (k-1) \pi / 2) & : \quad k=1,2,3,4 \\ \sqrt{2}(\cos (k-5) \pi / 2+\pi / 4, \sin (k-5) \pi / 2+\pi / 4) & : \quad k=5,6,7,8\end{cases}
$$

$\mathbf{S}=\operatorname{diag}(1,0.2,0.1,1,1.2,1,1.2,1 / \tau, 1 / \tau)$ and the transform matrix $\mathbf{M}$ reads

$$
\mathbf{M}=\left(\begin{array}{ccccccccc}
1 & 1 & 1 & 1 & 1 & 1 & 1 & 1 & 1 \\
-4 & -1 & -1 & -1 & -1 & 2 & 2 & 2 & 2 \\
4 & -2 & -2 & -2 & -2 & 1 & 1 & 1 & 1 \\
0 & 1 & 0 & -1 & 0 & 1 & -1 & -1 & 1 \\
0 & -2 & 0 & 2 & 0 & 1 & -1 & -1 & 1 \\
0 & 0 & 1 & 0 & -1 & 1 & 1 & -1 & -1 \\
0 & 0 & -2 & 0 & 2 & 1 & 1 & -1 & -1 \\
0 & 1 & -1 & 1 & -1 & 0 & 0 & 0 & 0 \\
0 & 0 & 0 & 0 & 0 & 1 & -1 & 1 & -1
\end{array}\right)
$$


and

$$
g_{k}^{(e q)}=\omega_{k} \rho\left[1+\frac{c \vec{e}_{k} \cdot \vec{v}}{c_{s}^{2}}+\frac{\left(c \vec{e}_{k} \cdot \vec{v}\right)^{2}}{2 c_{s}^{4}}-\frac{|\vec{v}|^{2}}{2 c_{s}^{2}}\right]
$$

where $\omega_{0}=4 / 9, \omega_{1 \sim 4}=1 / 9$ and $\omega_{5 \sim 8}=1 / 36 . c_{s}=c / \sqrt{3}$ is the sound speed. For consistency, the forcing terms $F_{k}$ should be given by

$$
F_{k}=\mathbf{M}^{-1}\left(\mathbf{I}-\frac{1}{2} \mathbf{S}\right) \mathbf{M}_{j k} \bar{F}_{k}
$$

where

$$
\bar{F}_{k}=\omega_{k}\left[\frac{c \vec{e}_{k} \cdot \vec{F}}{c_{s}^{2}}+\frac{\left(c \vec{e}_{k} \cdot \vec{v}\right)\left(c \vec{e}_{k} \cdot \vec{F}\right)}{c_{s}^{4}}-\frac{\vec{v} \cdot \vec{F}}{c_{s}^{2}}\right]
$$

and $\mathbf{I}$ is the unity matrix. The velocity $\vec{v}$ is defined as

$$
\vec{v}=\sum_{k \geq 0} c \vec{e}_{k} g_{k}+\frac{\Delta t}{2} \vec{F}
$$

where

$$
\vec{F} \equiv \operatorname{Pr}(T-N Y) \frac{\vec{g}}{|\vec{g}|}
$$

The effective kinematic viscosity is determined by

$$
\nu_{e}=(\tau-0.5) c_{s}^{2} \Delta t
$$

and $\nu_{e}$ can be split into two parts:

$$
\nu_{e}=\nu_{0}+\nu_{t}
$$

where $\nu_{0}=\operatorname{Pr} R a^{-0.5}$ is the initial kinetic viscosity, and the turbulent eddy viscosity $\nu_{t}$ is obtained by

$$
\nu_{t}=(C \Delta)^{2}\left(|\bar{\phi}|^{2}+\frac{P r}{P r_{t}} \nabla(T-N Y) \cdot \frac{\vec{g}}{|\vec{g}|}\right)^{1 / 2}
$$

In the above equation, the constant $C$ is the so-called Smagorinsky constant and $\operatorname{Pr}_{t}$ represents the turbulent Prandtl number. $\Delta$ is the filter width. $|\bar{\phi}|$ is 
the magnitude of the large scale strain rate tensor

$$
|\bar{\phi}|=\sqrt{2 \bar{\phi}_{\alpha \beta} \bar{\phi}_{\alpha \beta}}
$$

where $\bar{\phi}_{\alpha \beta}=\left(\partial_{\alpha} \bar{u}_{\beta}+\partial_{\beta} \bar{u}_{\alpha}\right) / 2$, and the over bar indicates filtered values. As shown by Ref.[15], after some tedious algebraic operations one can get:

$$
\tau=\tau_{0}+\frac{(C \Delta)^{2}}{c_{s}^{2} \Delta t}\left(|\phi|^{2}+\frac{P r}{P r_{t}} \nabla(T-N Y) \cdot \frac{\vec{g}}{|\vec{g}|}\right)^{1 / 2}
$$

where $\tau_{0}=\nu_{0} /\left(c_{s}^{2} \Delta t\right)+0.5$.

The evolution equation for the temperature field reads

$$
f_{j}\left(\vec{x}+c \vec{e}_{j} \Delta t, t+\Delta t\right)-f_{j}(\vec{x}, t)=-\tau_{\Theta}^{-1}\left[f_{j}(\vec{x}, t)-f_{j}^{(e q)}(\vec{x}, t)\right]
$$

where $\tau_{\Theta}$ is the dimensionless relaxation time for temperature field and

$$
f_{j}^{(e q)}=\frac{T}{b}\left[1+b \frac{\vec{e}_{j} \cdot \vec{u}}{2 c}\right]
$$

where $b$ is the number of discrete velocity directions for the temperature field [15]. The temperature $T$ is obtained in terms of the distribution function by

$$
T=\sum_{j} f_{j}
$$

The effective thermal diffusivity $\alpha_{e}$ is given by

$$
\alpha_{e}=2 c^{2}\left(\tau_{\Theta}-0.5\right) \Delta t / b
$$

Similar as $\nu_{e}$, the effective thermal diffusivity $\alpha_{e}$ also can be split into two parts:

$$
\alpha_{e}=\alpha_{0}+\alpha_{t}
$$

$\alpha_{0}=R a^{-0.5}$ is the initial thermal diffusivity. The turbulent thermal diffusivity $\alpha_{t}=\nu_{t} / \operatorname{Pr}_{t}$. And

$$
\tau_{\Theta}=\tau_{\Theta 0}+\frac{b D_{t}}{2 c^{2} \Delta t}
$$


where $\tau_{\Theta 0}=b \alpha_{0} /\left(2 c^{2} \Delta t\right)+0.5$.

The evolution equation for the concentration field reads

$$
h_{j}\left(\vec{x}+c \vec{e}_{j} \Delta t, t+\Delta t\right)-h_{j}(\vec{x}, t)=-\tau_{D}^{-1}\left[h_{j}(\vec{x}, t)-h_{j}^{(e q)}(\vec{x}, t)\right]
$$

where $\tau_{D}$ is the dimensionless relaxation time for concentration field and

$$
h_{j}^{(e q)}=\frac{Y}{b}\left[1+b \frac{\vec{e}_{j} \cdot \vec{u}}{2 c}\right]
$$

where $b$ is the number of discrete velocity directions for the concentration field [15]. The concentration $Y$ is obtained in terms of the distribution function by

$$
Y=\sum_{j} h_{j}
$$

The effective solutal diffusivity $D_{e}$ is given by

$$
D_{e}=2 c^{2}\left(\tau_{D}-0.5\right) \Delta t / b
$$

The effective solutal diffusivity $D_{e}$ also can be split into two parts:

$$
D_{e}=D_{0}+D_{t}
$$

$D_{0}=L e R a^{-0.5}$ is the initial solutal diffusivity, where Le is the Lewis number. The turbulent solutal diffusivity $D_{t}=\nu_{t} / S c_{t}$, where $S c_{t}$ is the turbulent Schmidt number. Consequently

$$
\tau_{D}=\tau_{D 0}+\frac{b D_{t}}{2 c^{2} \Delta t}
$$

where $\tau_{D 0}=b D_{0} /\left(2 c^{2} \Delta t\right)+0.5$.

The same as that in Ref.[15], in the present study, we employ the D2Q5 lattice model to solve the scalar fields, namely $b=5$ in Eqs.(19) and (25). The values of $C, P r_{t}$ and $S c_{t}$ are identical with that in Ref.[15], too. 


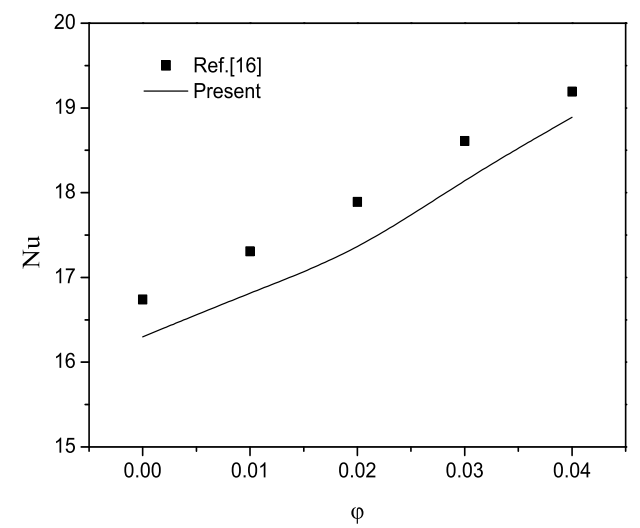

Fig. 2. Comparison of the Nusselt number for various nanoparticle volume fraction when $R a=10^{7}$.

\section{Numerical validation}

The reliability of the present LB-LES approach for turbulent double diffusion simulation has been demonstrated in Ref.[15], where the Rayleigh number varies from $10^{7}$ to $10^{11}$. Therefore, in this section we only validate its applicability for nanofluid. For this purpose, natural convection of water- $\mathrm{SiO}_{2}$ nanofluid in a square enclosure proposed in Ref.[18] is adopted. Figure 2 illustrates the variation of the averaged Nusselt number $(N u)$ with the nanoparticle volume fraction $(\varphi)$ when $R a=10^{7}$. The predictions obtained by the present approach agree well with that in Ref. [18], which demonstrates the accuracy of our numerical approach for nanofluid simulation.

\section{$6 \quad$ Results and discussions}

In the present study, we simulate double-diffusive convection of water- $\mathrm{SiO}_{2}$ nanofluid in a square cavity with $10^{5} \leq R a \leq 10^{10}$ and $0 \leq N \leq 2$. In Ref.[18] the physical properties of water- $\mathrm{SiO}_{2}$ nanofluid, which obtained through experimental measure, were provided only for $0 \leq \varphi \leq 0.04$, so this work also focuses on the same range. When $R a \leq 10^{9}$ the grid resolution $128 \times 128$ is employed while $256 \times 256$ for $R a=10^{10}$. Our previous study [15] has shown that such grid resolutions are fine enough to capture the sophisticated physical structures.

Figure 3-8 depict the isotherms, iso-concentrations and stream lines of doublediffusive convection of water- $\mathrm{SiO}_{2}$ nanofluid over a wide range, from laminar regimes $\left(R a<10^{7}\right)$ to fully turbulent regimes $\left(R a \geq 10^{9}\right)$. While $R a=10^{5}$ (Fig.3), the patterns of isotherms, iso-concentrations and stream lines are 
strictly symmetrical respect to the center of the square cavity. The isotherms and iso-concentrations are nearly vertical except in the vicinity of the center of the domain, which indicates the heat being transferred dominantly by conduction at low Rayleigh number. In addition, stable stratification of isotherms and iso-concentrations occurs near the vertical walls. There are two small vortices appear around the center of the enclosure. As the Rayleigh number increases,replacing conduction, convection becomes being the predominant heat transfer mechanism. Accordingly, the isotherms and iso-concentrations emerge horizontally in the cavity except in the neighbourhood of the boundaries (Fig.4). Especially, the symmetry begins to be broken even in the laminar regime $R a=10^{6}$, which can be illustrated very clearly by the plot of stream lines: a third vortex has formed in the core region and the two vortices surrounding it are not point symmetric any longer. In the transitional regimes $\left(10^{7} \leq R a \leq 10^{8}\right)$ (Figs.5-6), the isotherms and iso-concentrations become straighter in the core of the cavity and sharper near the vertical walls. The vortices expand quickly with the Rayleigh number enhancing. Albeit the isotherms and iso-concentrations seem to keep point symmetric still, the pattern of stream lines has lost its symmetry obviously: two large flow structures near the top-left corner and bottom-right corner are only similar with each other, rather than point symmetric. After a comparison between the flow field at $R a=10^{7}$ and that at $R a=10^{8}$, one can find that such similarity will be undermined further by the emerging fine irregular vortices at higher $R a$. The double diffusion natural convection becomes turbulent since $R a=10^{9}$. As plotted by Fig.7, there appears clear stratification of isotherms and iso-concentrations along the vertical direction within almost the whole domain except the very thin layers attached on the vertical walls. It is difficult to find large similar flow structures under this situation, except near the top-left corner and bottom-right corner. When $R a$ reaches $10^{10}$ (Fig.8), the double diffusion becomes complete chaos. The enclosure is full of small-scale flow structures. Even the stratification of isotherms and iso-concentrations has been destroyed by them, especially near the corners. As shown by these plots, for double diffusion natural convection of nanofluid within a square cavity, the patterns of isotherms are always identical to that of iso-concentrations, in spite of whatever $R a$ being.

Figure 9 shows the averaged temperature and velocity profiles at the midsections of the cavity for various $R a$. These profiles form some waviness. It is clear that against $R a$ increasing, the temperature difference in the core of the domain becomes diminishing. Since $R a>10^{7}$, the temperature profiles within the core region of the cavity are nearly overlapped with each other. Only in the laminar regime $R a=10^{5}$, there is sufficient temperature gradient to maintain heat conduction in the center of the enclosure. Then the temperature "waves" move rapidly towards the boundaries and are flattened with ascending $R a$ until $R a=10^{9}$. In the turbulent regimes $\left(R a \geq 10^{9}\right)$, the temperature "peaks" begin to rise with $R a$ increasing, which indicates again the 


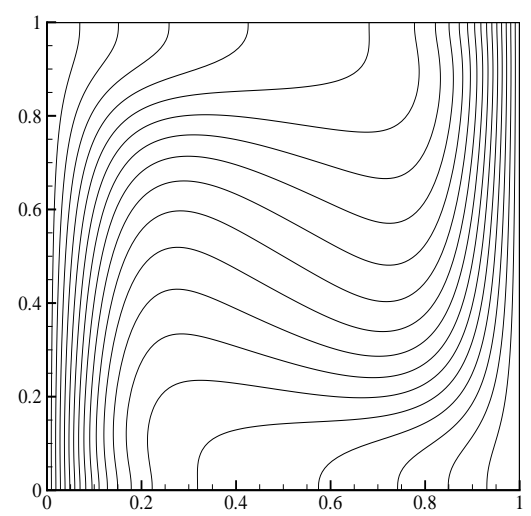

(a)

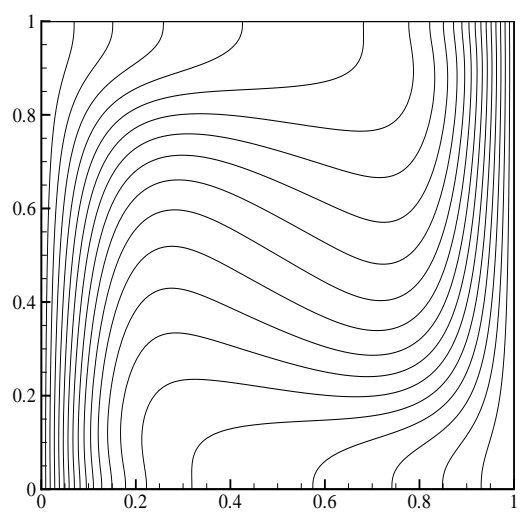

(b)

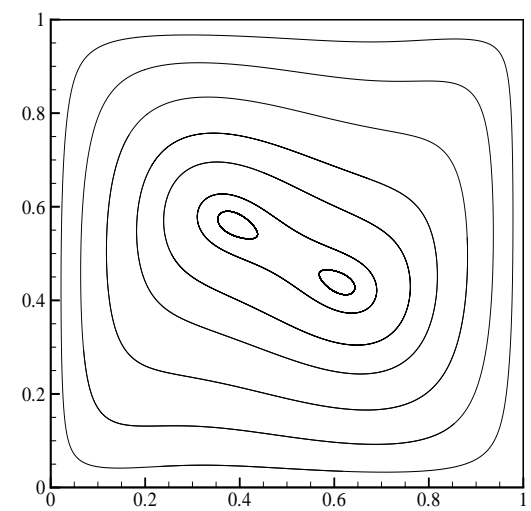

(c)

Fig. 3. (a) isotherms (b) iso-concentration and (c) stream lines for $R a=10^{5}$, $\varphi=0.04$ and $N=0.5$.

heat transfer mechanism of double diffusion of nanofluid in turbulent regimes is quite different from that in laminar regimes. In the turbulent regimes, heat conduction predominates just within the very thin layers attached with the vertical walls and heat transfer becomes more intensively in the boundary layers with $R a$ increasing. The vertical velocity profiles also can demonstrate such transition. Here we must correct one conclusion drawn in a previous publication [9]. In Ref.[9], the authors claimed that nanofluid would move with greater velocity for a higher $R a$ while the waviness would decrease for lower values of Ra. According to Fig. 9 (b), it is clear that such conclusion is questionable. The peak vertical velocity at $R a=10^{7}$ is obviously lower than that at $R a=10^{6}$, although within the transitional regimes $\left(10^{7} \leq R a \leq 10^{8}\right)$ the maximum value of vertical velocity ascends slightly with $R a$. Especially, as the double diffusion natural convection of nanofluid reaches the turbulent regimes $\left(R a \geq 10^{9}\right)$, the peak values of vertical velocity keep down against $R a$ increasing, which means nanofluid within the boundary layers will move more slowly at higher $R a$. Such unusual phenomena result from the combined effects of vertical solid walls and boundary layers. Therefore there is no u- 


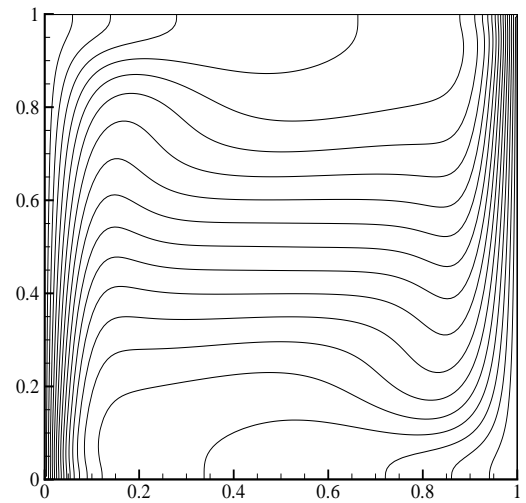

(a)

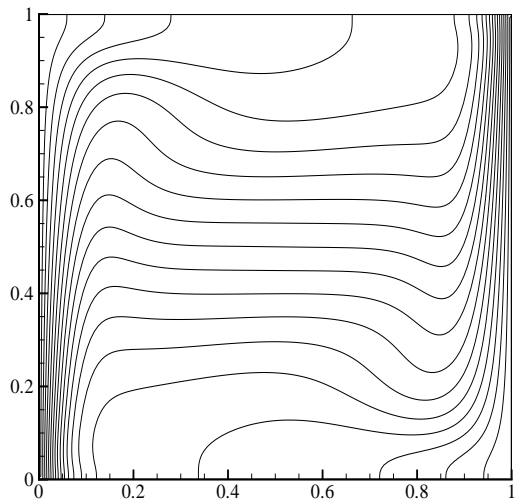

(b)

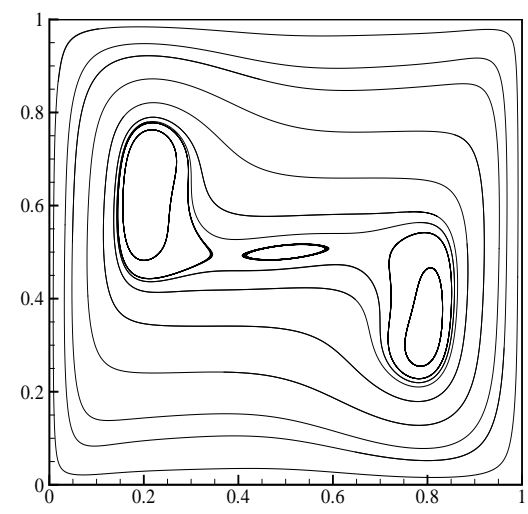

(c)

Fig. 4. (a) isotherms (b) iso-concentration and (c) stream lines for $R a=10^{6}$, $\varphi=0.04$ and $N=0.5$.

niversal characteristic over the whole range varying from laminar regimes to turbulent regimes except that the zone with obvious vertical movement of fluid is compressed quickly against $R a$ enhancing. Figure 9 (c), which plots the horizontal velocity profile, can further support our viewpoint. As shown by Fig. 9 (c), the maximum value of horizontal velocity diminishes monotonically against $R a$ and horizontal motion of working fluid becomes weakly along the mid-section. Taking the stream lines plotted in Figs. 3-8 into account, one can find that during the transition from laminar double diffusive convection of nanofluid to its turbulent counterpart, the regular large-scale circulation of working fluid will be replaced by numerous relatively lower speed irregular vortices scattered in the whole cavity. Consequently, intensive heat and mass transfer of nanofluid can take place almost across the whole domain with the aid of these irregular vortices, which can be demonstrated by Fig.10. Figure 10 illustrates the corresponding Nusselt number and Sherwood number. $\mathrm{Nu}$ and $S h$ both are monotonic increasing functions of $R a$. In the laminar regimes $\left(R a \leq 10^{6}\right), N u$ and $S h$ increase slowly with $R a$. Then they are enhanced more and more sharply, especially in the turbulent regimes $R a \geq 10^{9}$. Quite differ- 


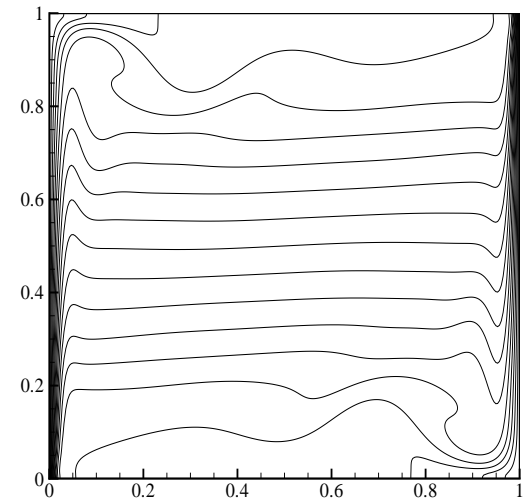

(a)

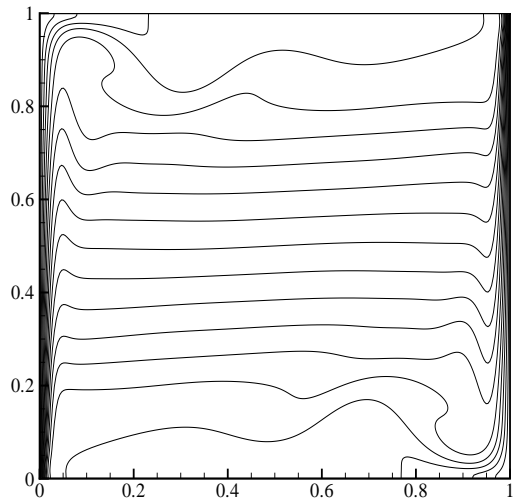

(b)

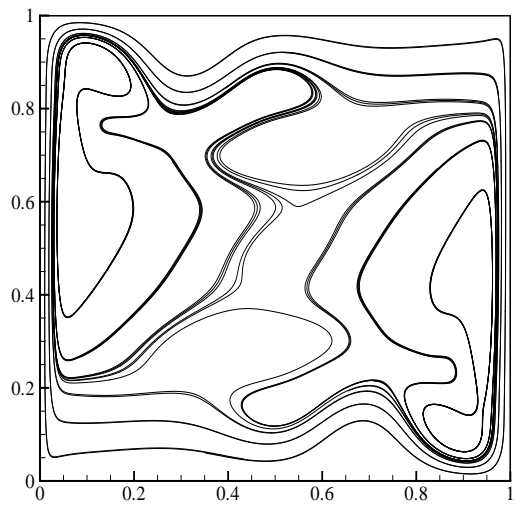

(c)

Fig. 5. (a) isotherms (b) iso-concentration and (c) stream lines for $R a=10^{7}$, $\varphi=0.04$ and $N=0.5$.

ent from the double diffusion in pure base fluid (air) [15], due to the existence of nanoparticle, the Sherwood number in the present investigated domain is always smaller than the Nusselt number. The difference between $N u$ and $S h$ is not apparent when $R a \leq 10^{6}$. However, the gap between them becomes more and more significant as $R a$ ascending. It implies heat transfer is enhanced more intensively than mass transfer at higher $R a$.

Figure 11-13 illustrate the averaged temperature and velocity profiles at the mid-sections of the cavity at $R a=10^{5}, R a=10^{7}$ and $R a=10^{9}$ with various $\varphi$. They are the representatives of the laminar regimes, transitional regimes and turbulent regimes, respectively. Although the profiles of vertical velocity and temperature both vary gradually with $\varphi$, the influences of nanoparticles on them are different. The increasing nanoparticle volume enhances the conductive properties of working fluid while suppresses some motion strength of base fluid near the vertical walls as the existence of nanoparticles can increase the effective thermal conductivity of the mixture as well as its effective viscosity. The profiles of horizontal velocity of nanofluid are changed slightly in the lam- 


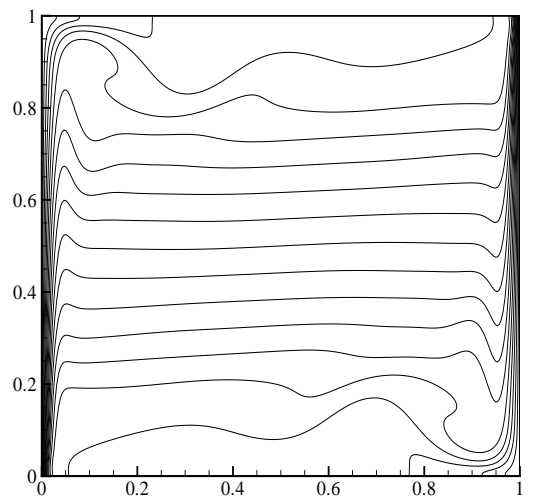

(a)

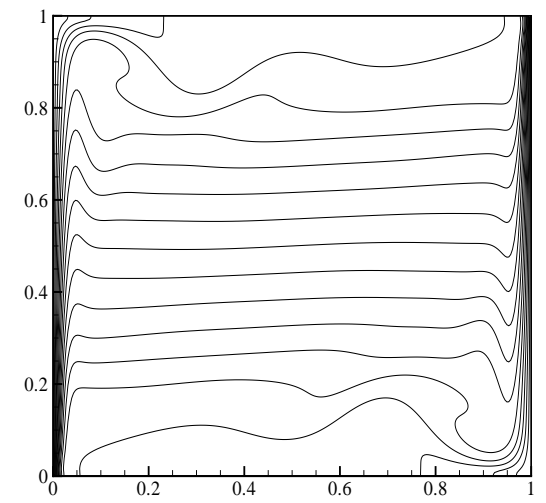

(b)

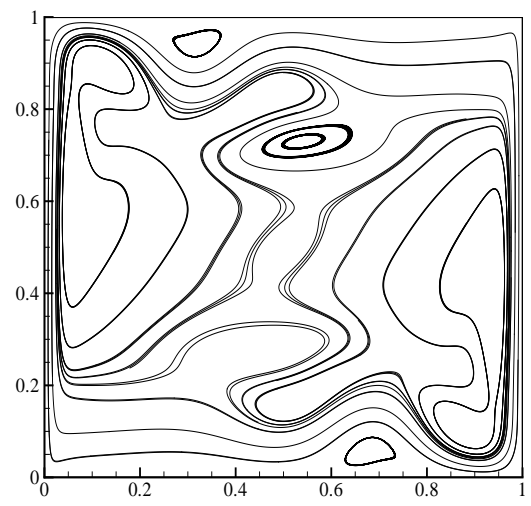

(c)

Fig. 6. (a) isotherms (b) iso-concentration and (c) stream lines for $R a=10^{8}$, $\varphi=0.04$ and $N=0.5$.

inar and transitional regimes while significantly in the turbulent regimes when more nanoparticles are added into the working fluid. In the laminar regimes, the movement of nanofluid near the center of the cavity is enhanced with increasing $\varphi$. However, beyond the laminar regimes, the motion of nanofluid in the vicinity of the core region is suppressed against $\varphi$ ascending. Although they seem very complicated and irregular, the phenomena can be explained by the change of effective viscosity of nanofluid in a universal way. It is wellknown that the viscosity of fluid always tries to diminish velocity gradients in flow. To show clearly the role of effective viscosity of nanofluid, we should ask the aid from the stream lines plotted in Figs. 3-8. When $R a=10^{5}$, there is only one large circulation in the cavity, so there are significant velocity differences along both the vertical and the horizontal direction at the mid-section. Nanofluid with a higher effective viscosity (corresponding to a bigger $\varphi$ ) can reduce the velocity differences more effectively. The corresponding phenomena are to flatten the velocity "waviness" more obviously by relative higher speed circulation in the vicinity of the core, as depicted by Fig. 11(a)-(b). While beyond the laminar regimes, the flow splits into two or more relative large-scale 


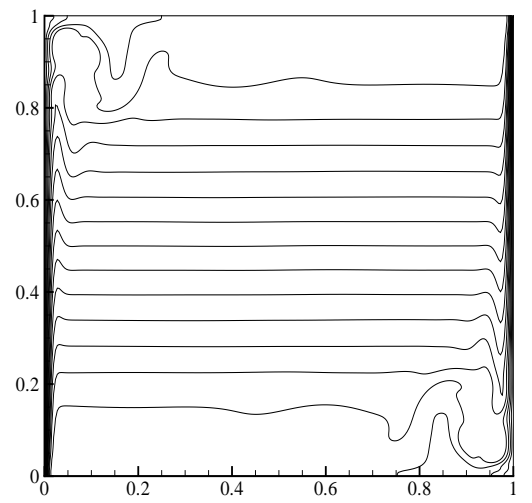

(a)

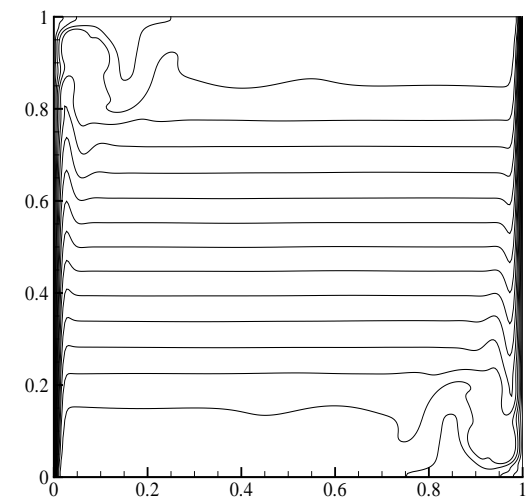

(b)

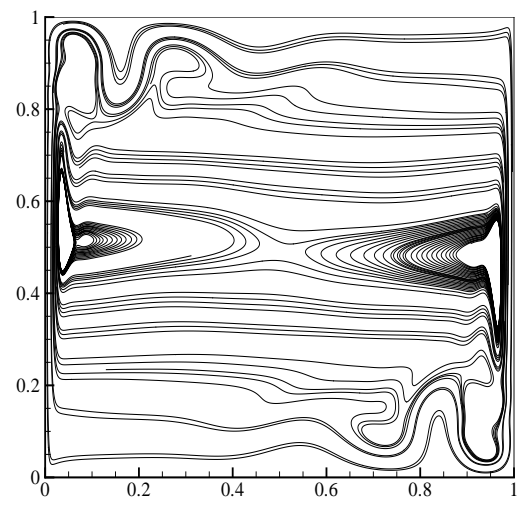

(c)

Fig. 7. (a) isotherms (b) iso-concentration and (c) stream lines for $R a=10^{9}$, $\varphi=0.04$ and $N=0.5$.

vortices (e.g. two large vortices at $R a=10^{7}$ ). Accordingly, the mid-section becomes the interface of these vortices, where fluid tries to keep stationary. Consequently, nanofluid with a higher effective viscosity will hamper the motion of flow in this zone more obviously. The corresponding phenomena are to flatten the velocity "waviness" through relative lower speed motion, as illustrated by Fig. 12(a)-(b). Furthermore, Fig. 13(a) implies the flow pattern of double diffusion of nanofluid in turbulent regimes is sensitive to $\varphi$ when nanoparticle load is small.

Figure 14 depicts the variation of the Nusselt and Sherwood number with different nanoparticle volume fraction. In Ref.[8], the authors found in laminar diffusion convection of nanofluid the Nusselt number would increase with $\varphi$ while the Sherwood number would decrease against $\varphi$. Our results demonstrate this conclusion is still valid beyond laminar regimes. Moreover, the influences of addition of nanoparticles on heat and mass transfer are clearer at higher $R a$ : when $R a=10^{5}$, the variation of $N u$ and $S h$ is slight while it becomes significant at $R a=10^{9}$. Moreover, over the whole range investigated in the 


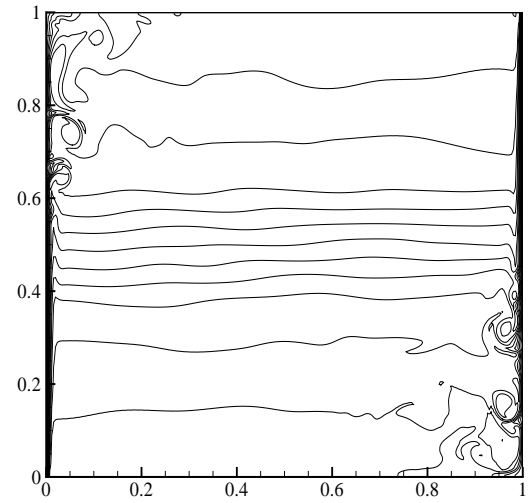

(a)

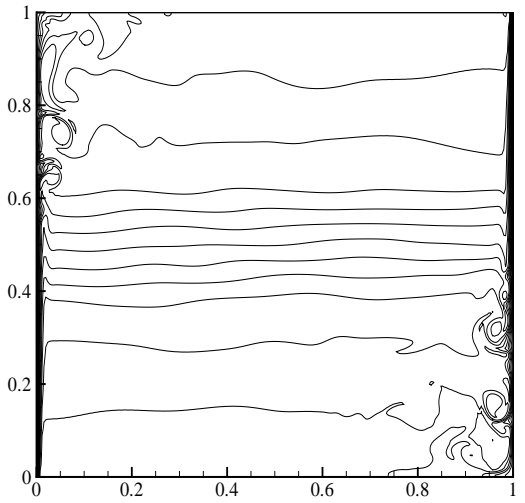

(b)

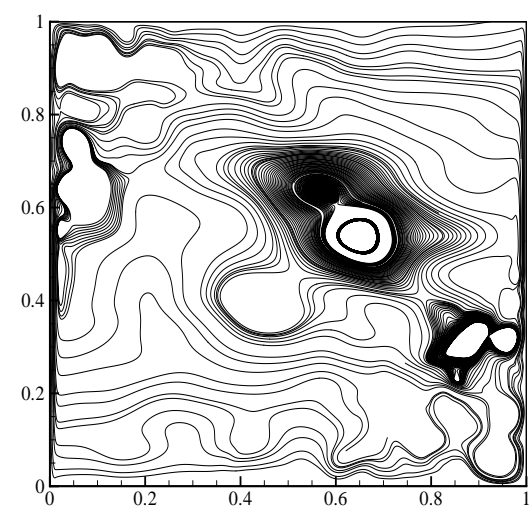

(c)

Fig. 8. (a) isotherms (b) iso-concentration and (c) stream lines for $R a=10^{10}$, $\varphi=0.04$ and $N=0.5$.

present work, $N u$ and $S h$ vary nearly linearly with $\varphi$. Comparison with the enhancement of addition of nanoparticles on heat transfer, the attenuation on concentration diffusion by nanoparticles is relatively weak.

In order to clearly show the effects of the ratio of buoyancy forces on double diffusion of nanofluid, we choose $R a=10^{5}$ as the representative. Figure 15 depicts the isotherms, iso-concentrations and stream lines when $R a=10^{5}$, $\varphi=0.04$ and $N=1.5$. It is clear that Fig. 15 is a mirror of Fig. 3 where $N=0.5$. When $N<1$, the thermal buoyancy is stronger than the compositional buoyancy and dominates the direction of motion of convection, so the circulation is clockwise. And vice versa, as $N>1$, the compositional buoyancy outweighs its thermal counterpart and consequently the circulation is counterclockwise. It is more clear with the aid of Fig.16, in which the corresponding vertical and horizontal velocity at the mid-sections of the cavity are presented. In addition, as $N$ approaches unity, the motion of nanofluid is being suppressed because the driven force, which results from the difference between the compositional buoyancy and the thermal buoyancy, becomes vanishing. No 


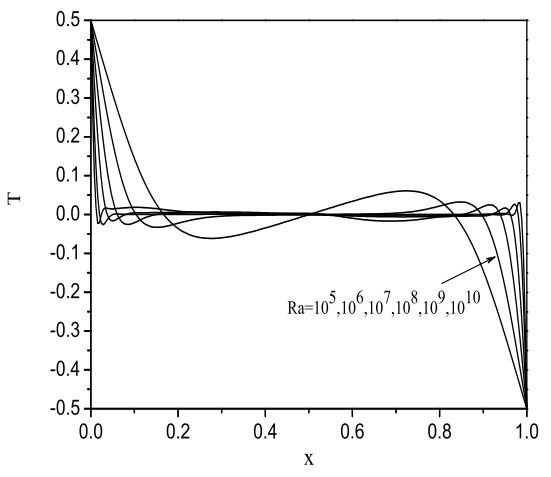

(a)

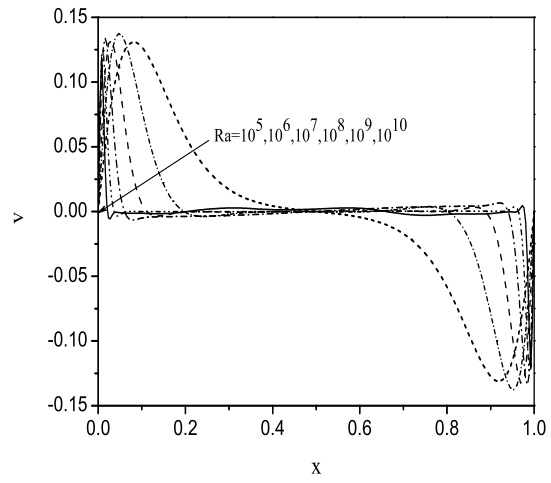

(b)

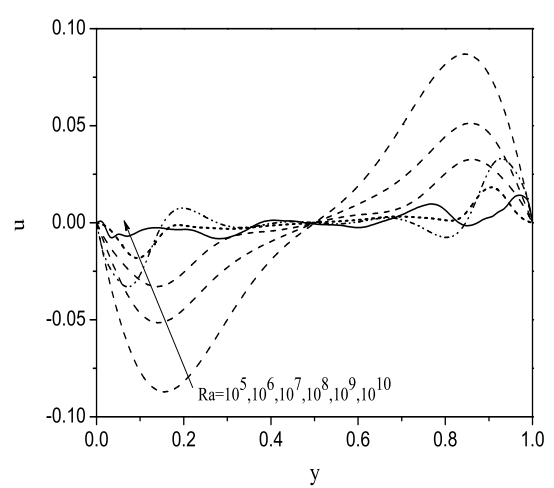

(c)

Fig. 9. Distributions of (a) temperature (b) vertical velocity and (c) horizontal velocity at the mid-sections of the cavity for various $R a$ with $\varphi=0.04$ and $N=0.5$.

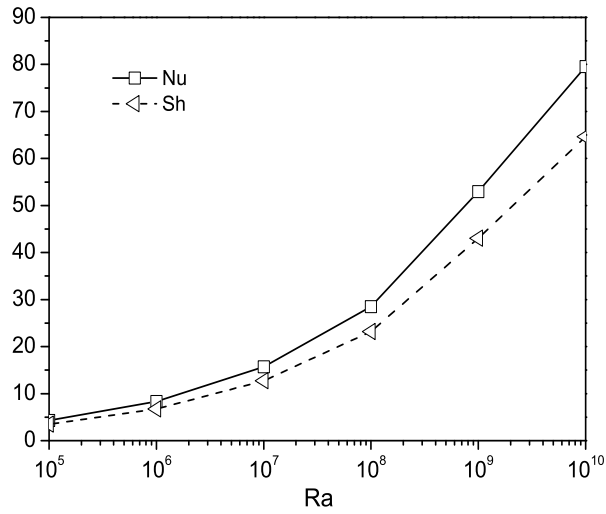

Fig. 10. Variation of $N u$ and $S h$ for various $R a$ when $\varphi=0.04$ and $N=0.5$. 


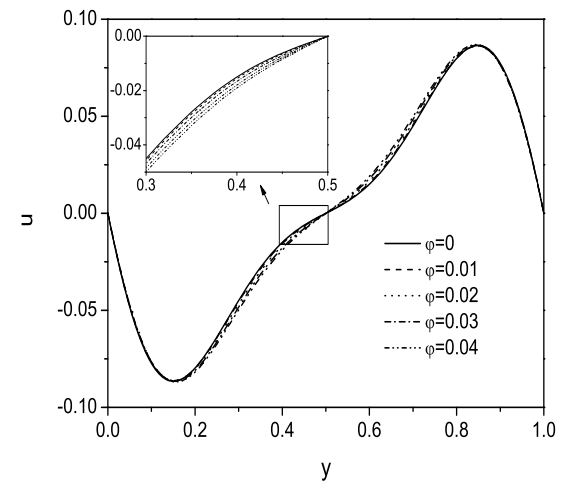

(a)

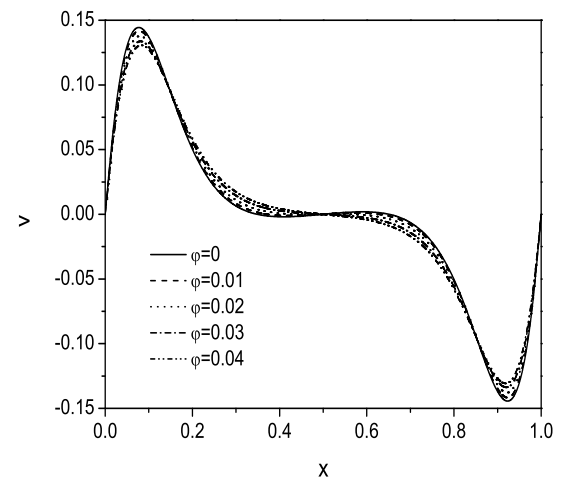

(b)

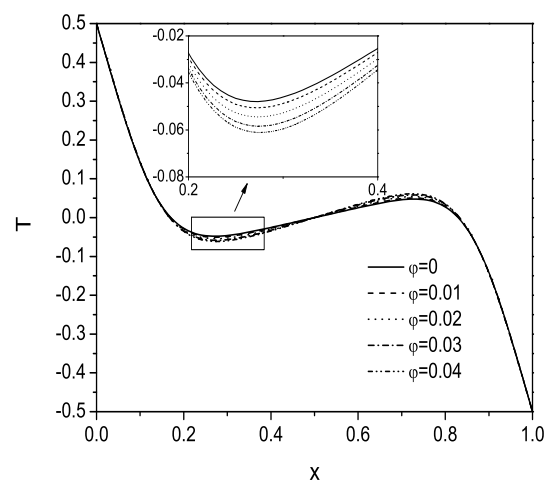

(c)

Fig. 11. Profile of (a) vertical velocity (b) horizontal velocity and (c) temperature at the mid-sections of the cavity for various $\varphi$ with $R a=10^{5}$ and $N=0.5$.

doubt, heat and mass transfer of double diffusion of nanofluid will be weakened when $N$ approaches unity, as illustrated by Fig.17. Another interesting result revealed by this figure is that $N u$ and $S h$ in laminar double diffusion of nanofluid will not be zero even when $N=1$, which is quite different from the numerical predictions for pure base fluid (air) [19]. We will discuss it in detail below.

Table (1)-(12) list the computed average Nusselt number and Sherwood number in various $R a, N$ and $\varphi$. According to these data, one can find that the Sherwood number keeps constant for all $\varphi$ when $N=1.0$ at a given $R a$ although the Nusselt number increases very slightly with $\varphi$ when $N=1.0$ at a given $R a$. When $R a \leq 10^{7}$, the minimums of $N u$ at a given $\varphi$ are identical. Moreover, it can be observed that the lowest values of $S h$ are 1.21 for almost all cases when $R a \leq 10^{7}$. The similar phenomena repeat when $R a \geq 10^{9}$. Beyond the laminar regimes, $N u$ and $S h$ both are bigger than zero when $N=1$, which is consistent with the discovery in Ref.[15,20]. Through a standard data fitting process, the following power-like correlation among $\mathrm{Nu} / \mathrm{Sh}, \mathrm{Ra}, \mathrm{N}$ and 


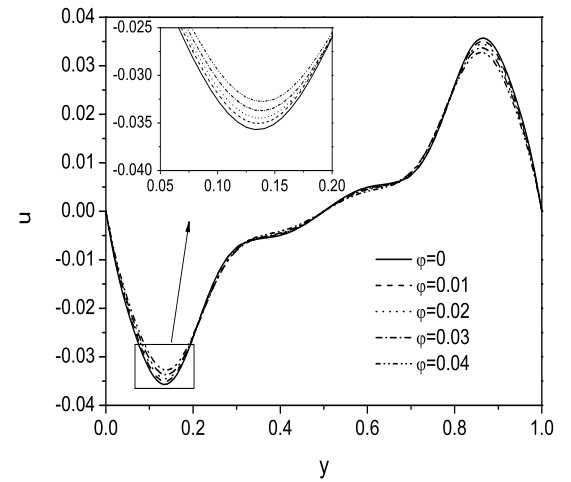

(a)

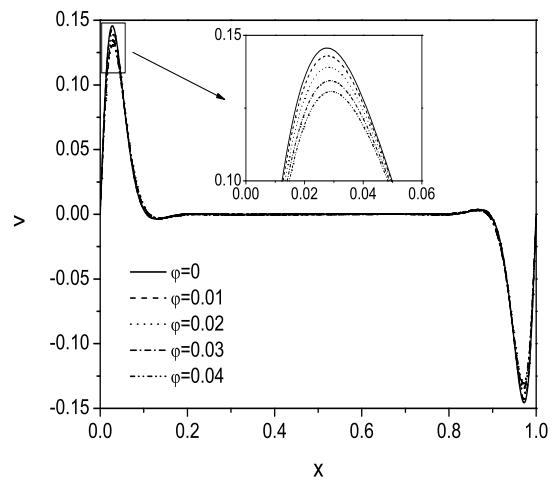

(b)

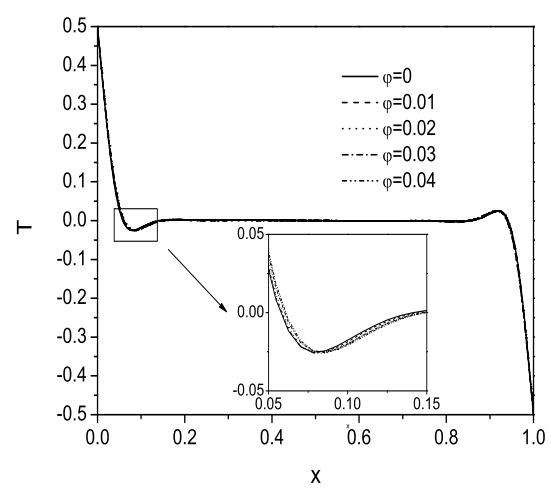

(c)

Fig. 12. Profile of (a) vertical velocity (b) horizontal velocity and (c) temperature at the mid-sections of the cavity for various $\varphi$ with $R a=10^{7}$ and $N=0.5$.

$\varphi$ can be extracted:

Table 1

The computed average Nusselt number at the hot wall when $R a=10^{5}$.

\begin{tabular}{cccccc}
\hline & $N=0$ & $N=0.5$ & $N=1.0$ & $N=1.5$ & $N=2.0$ \\
\hline$\varphi=0$ & 4.31 & 3.65 & 1.20 & 3.65 & 4.31 \\
$\varphi=0.01$ & 4.39 & 3.74 & 1.25 & 3.74 & 4.39 \\
$\varphi=0.02$ & 4.54 & 3.91 & 1.32 & 3.91 & 4.54 \\
$\varphi=0.03$ & 4.76 & 4.13 & 1.41 & 4.13 & 4.76 \\
$\varphi=0.04$ & 4.94 & 4.31 & 1.49 & 4.31 & 4.94 \\
\hline
\end{tabular}

$$
N u=a \times(1+b \times \varphi) \times(R a \times|1-N|)^{c}+d \times \varphi+e
$$

and

$$
S h=\mathfrak{a} \times(1-\mathfrak{b} \times \varphi) \times(R a \times|1-N|)^{\mathfrak{c}}+\mathfrak{d}
$$




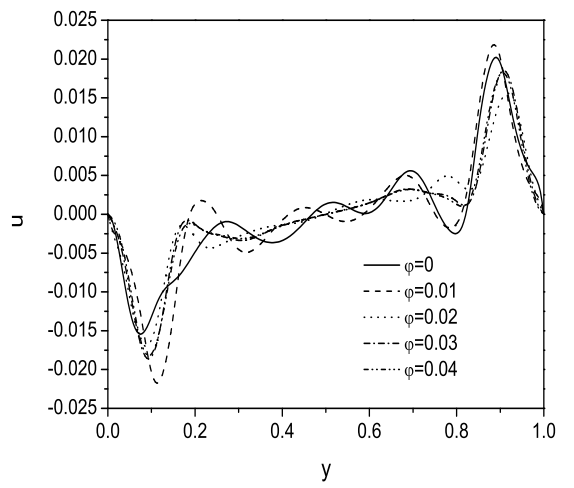

(a)

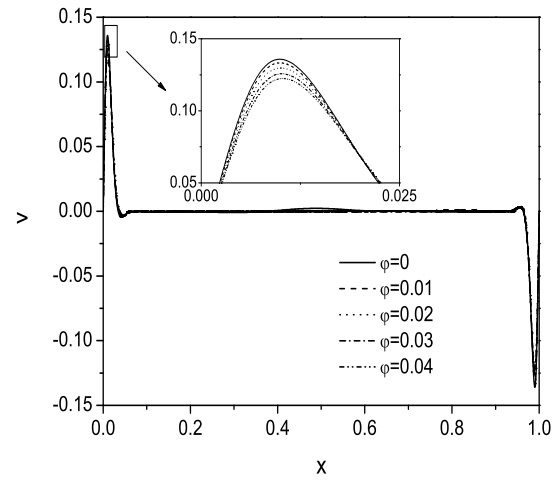

(b)

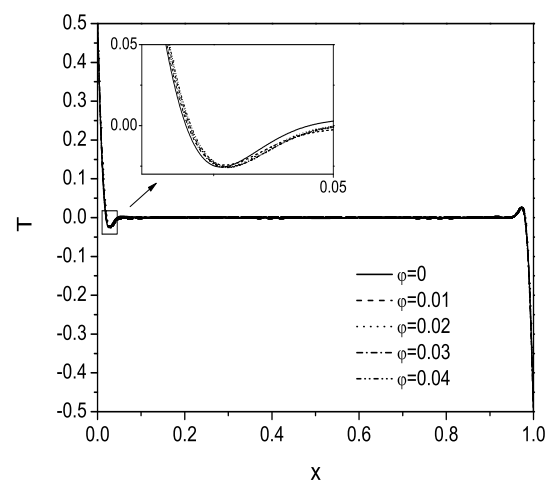

(c)

Fig. 13. Profile of (a) vertical velocity (b) horizontal velocity and (c) temperature at the mid-sections of the cavity for various $\varphi$ with $R a=10^{9}$ and $N=0.5$.

Table 2

The computed average Sherwood number at the hot wall when $R a=10^{5}$.

\begin{tabular}{cccccc}
\hline & $N=0$ & $N=0.5$ & $N=1.0$ & $N=1.5$ & $N=2.0$ \\
\hline$\varphi=0$ & 4.31 & 3.65 & 1.20 & 3.65 & 4.31 \\
$\varphi=0.01$ & 4.25 & 3.63 & 1.21 & 3.63 & 4.25 \\
$\varphi=0.02$ & 4.17 & 3.59 & 1.21 & 3.59 & 4.17 \\
$\varphi=0.03$ & 4.08 & 3.54 & 1.21 & 3.54 & 4.08 \\
$\varphi=0.04$ & 4.02 & 3.50 & 1.21 & 3.50 & 4.02 \\
\hline
\end{tabular}

The coefficients in the above two equations have been listed in Table 13-14. One can find the coefficients vary slightly when $R a<10^{7}$, which implies that in the laminar regimes, the heat and mass transfer mechanism does not change significantly. 


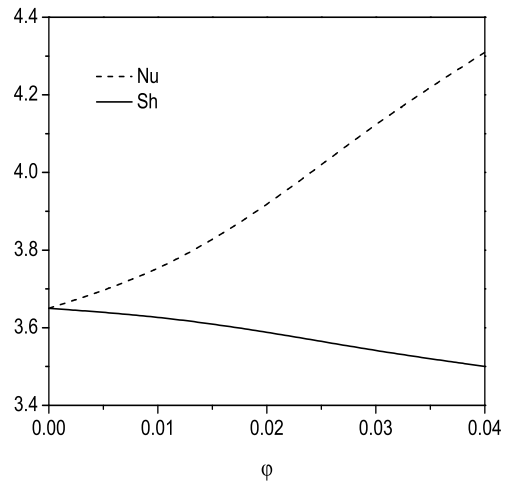

(a)

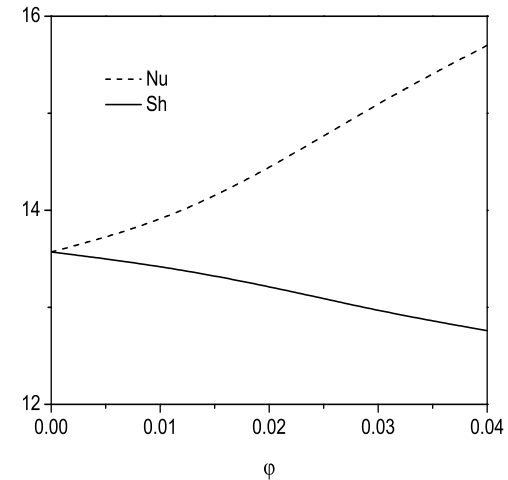

(b)

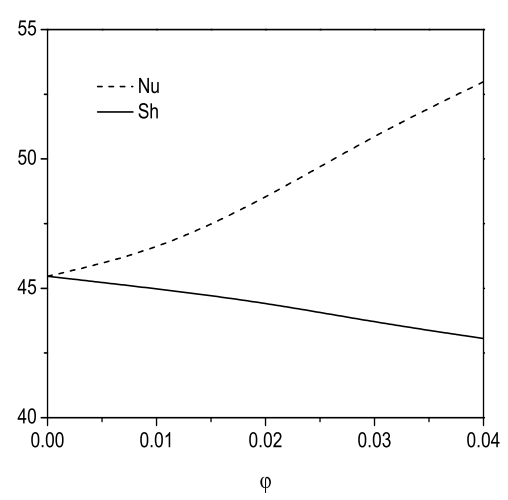

(c)

Fig. 14. Variation of $N u$ and $S h$ for various $\varphi$ with $N=0.5$ and (a) $R a=10^{5}$ (b) $R a=10^{7}$ and (c) $R a=10^{9}$.

Table 3

The computed average Nusselt number at the hot wall when $R a=10^{6}$.

\begin{tabular}{cccccc}
\hline & $N=0$ & $N=0.5$ & $N=1.0$ & $N=1.5$ & $N=2.0$ \\
\hline$\varphi=0$ & 8.77 & 7.16 & 1.21 & 7.16 & 8.73 \\
$\varphi=0.01$ & 8.95 & 7.32 & 1.25 & 7.32 & 8.91 \\
$\varphi=0.02$ & 9.29 & 7.62 & 1.32 & 7.62 & 9.25 \\
$\varphi=0.03$ & 9.73 & 7.99 & 1.41 & 7.99 & 9.71 \\
$\varphi=0.04$ & 10.11 & 8.31 & 1.49 & 8.31 & 10.10 \\
\hline
\end{tabular}

\section{Conclusion}

Double diffusive natural convection of nanofluid is commonly found in renewable energy industry. However, the related studies on its fundamental characteristics are quite sparse, especially beyond the laminar regimes. As emphasized in the latest review paper [4], the insight into the performance of 


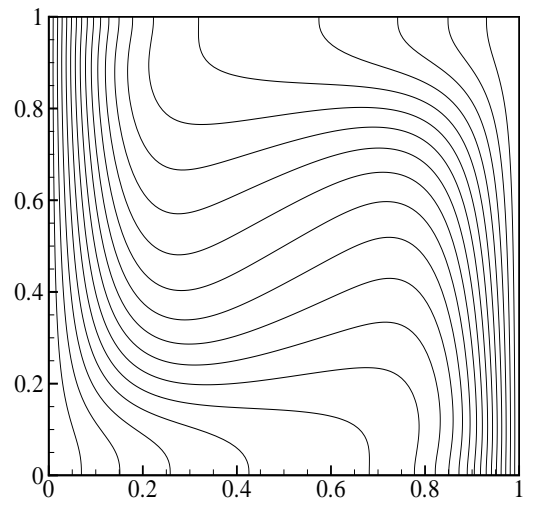

(a)

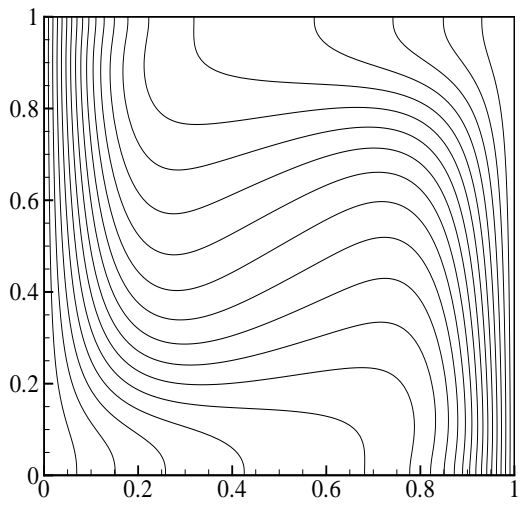

(b)

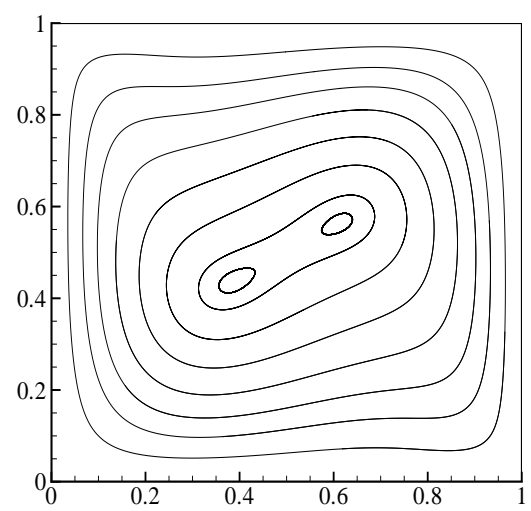

(c)

Fig. 15. (a) isotherms (b) iso-concentration and (c) stream lines for $R a=10^{5}$, $\varphi=0.04$ and $N=1.5$.

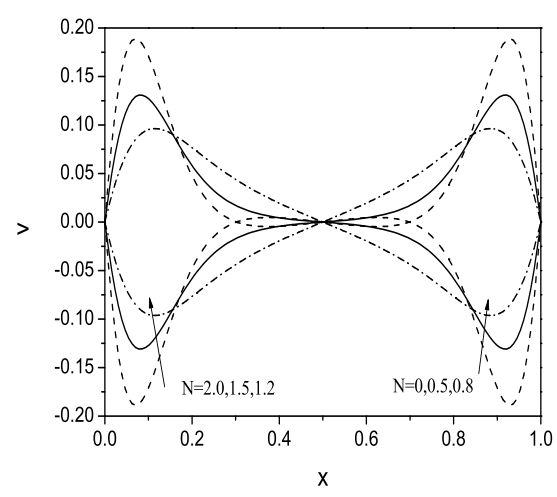

(a)

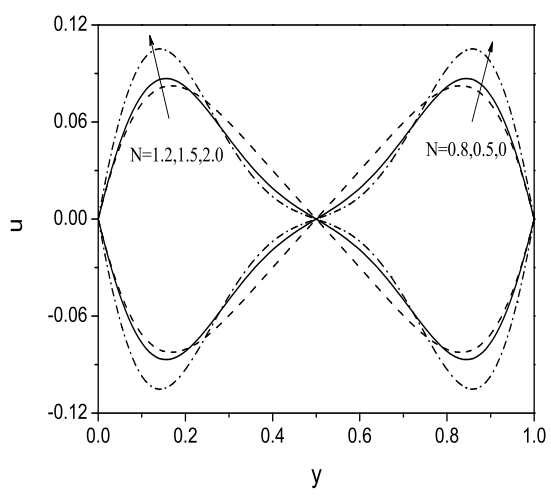

(b)

Fig. 16. Distributions of (a) vertical velocity and (b) horizontal velocity at the mid-sections of the cavity for various $N$ with $\varphi=0.04$ and $R a=10^{5}$. 


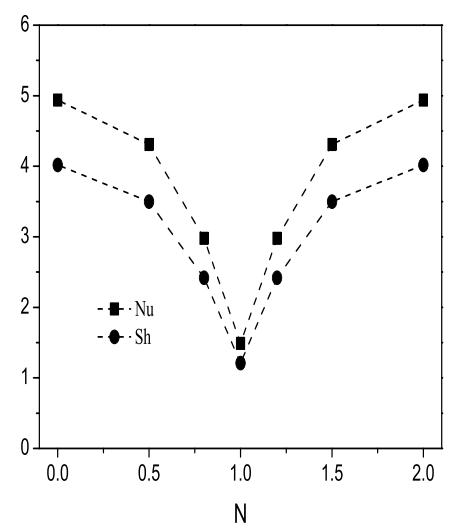

Fig. 17. Variation of the Nusselt number and Sherwood number for various $N$ with $\varphi=0.04$ and $R a=10^{5}$.

Table 4

The computed average Sherwood number at the hot wall when $R a=10^{6}$.

\begin{tabular}{cccccc}
\hline & $N=0$ & $N=0.5$ & $N=1.0$ & $N=1.5$ & $N=2.0$ \\
\hline$\varphi=0$ & 8.77 & 7.16 & 1.21 & 7.16 & 8.73 \\
$\varphi=0.01$ & 8.67 & 7.10 & 1.21 & 7.10 & 8.63 \\
$\varphi=0.02$ & 8.52 & 6.99 & 1.21 & 6.99 & 8.48 \\
$\varphi=0.03$ & 8.35 & 6.86 & 1.21 & 6.86 & 8.33 \\
$\varphi=0.04$ & 8.22 & 6.75 & 1.21 & 6.75 & 8.21 \\
\hline
\end{tabular}

Table 5

The computed average Nusselt number at the hot wall when $R a=10^{7}$.

\begin{tabular}{cccccc}
\hline & $N=0$ & $N=0.5$ & $N=1.0$ & $N=1.5$ & $N=2.0$ \\
\hline$\varphi=0$ & 16.30 & 13.57 & 1.21 & 13.57 & 16.30 \\
$\varphi=0.01$ & 16.65 & 13.87 & 1.25 & 13.87 & 16.65 \\
$\varphi=0.02$ & 17.30 & 14.42 & 1.32 & 14.42 & 17.32 \\
$\varphi=0.03$ & 18.16 & 15.11 & 1.41 & 15.11 & 18.16 \\
$\varphi=0.04$ & 18.89 & 15.70 & 1.49 & 15.70 & 18.89 \\
\hline
\end{tabular}

nanofluid in turbulent regimes arises a challenge not only in academic research but also in energy engineering. To deepen our understanding in this important area, the present work tries to reveal the heat and mass transfer mechanism of double diffusion of nanofluid over a quite broad range, from laminar regimes to turbulent regimes, for the first time. It is observed that the influences of addition of nanoparticles into base fluid are quite different between the laminar regimes and turbulent regimes. Some conclusions drawn from laminar double diffuiosn of nanofluid may be invalid in its turbulent counterparts. We try to explain these complicated phenomena through a universal way. In addition, the influence of the ratio of buoyancy forces on the performance of double dif- 
Table 6

The computed average Sherwood number at the hot wall when $R a=10^{7}$.

\begin{tabular}{cccccc}
\hline & $N=0$ & $N=0.5$ & $N=1.0$ & $N=1.5$ & $N=2.0$ \\
\hline$\varphi=0$ & 16.30 & 13.57 & 1.21 & 13.57 & 16.30 \\
$\varphi=0.01$ & 16.14 & 13.43 & 1.21 & 13.43 & 16.13 \\
$\varphi=0.02$ & 15.89 & 13.22 & 1.21 & 13.22 & 15.88 \\
$\varphi=0.03$ & 15.59 & 12.96 & 1.21 & 12.96 & 15.58 \\
$\varphi=0.04$ & 15.35 & 12.76 & 1.21 & 12.76 & 15.35
\end{tabular}

Table 7

The computed average Nusselt number at the hot wall when $R a=10^{8}$.

\begin{tabular}{cccccc}
\hline & $N=0$ & $N=0.5$ & $N=1.0$ & $N=1.5$ & $N=2.0$ \\
\hline$\varphi=0$ & 30.18 & 24.88 & 2.36 & 24.88 & 29.88 \\
$\varphi=0.01$ & 30.92 & 25.55 & 2.44 & 25.55 & 30.71 \\
$\varphi=0.02$ & 32.20 & 26.47 & 2.58 & 26.47 & 32.08 \\
$\varphi=0.03$ & 33.63 & 27.39 & 2.75 & 27.39 & 32.23 \\
$\varphi=0.04$ & 35.16 & 28.51 & 2.91 & 28.51 & 34.74 \\
\hline
\end{tabular}

Table 8

The computed average Sherwood number at the hot wall when $R a=10^{8}$.

\begin{tabular}{cccccc}
\hline & $N=0$ & $N=0.5$ & $N=1.0$ & $N=1.5$ & $N=2.0$ \\
\hline$\varphi=0$ & 30.18 & 24.88 & 2.36 & 24.88 & 29.88 \\
$\varphi=0.01$ & 29.95 & 24.75 & 2.36 & 24.75 & 29.75 \\
$\varphi=0.02$ & 29.53 & 24.27 & 2.36 & 24.27 & 29.41 \\
$\varphi=0.03$ & 28.85 & 23.50 & 2.36 & 23.49 & 28.51 \\
$\varphi=0.04$ & 28.58 & 23.17 & 2.36 & 23.17 & 28.24 \\
\hline
\end{tabular}

Table 9

The computed average Nusselt number at the hot wall when $R a=10^{9}$.

\begin{tabular}{cccccc}
\hline & $N=0$ & $N=0.5$ & $N=1.0$ & $N=1.5$ & $N=2.0$ \\
\hline$\varphi=0$ & 52.97 & 45.47 & 2.12 & 45.44 & 52.99 \\
$\varphi=0.01$ & 54.21 & 46.45 & 2.19 & 46.58 & 54.21 \\
$\varphi=0.02$ & 56.48 & 48.46 & 2.31 & 48.45 & 56.46 \\
$\varphi=0.03$ & 59.50 & 50.93 & 2.47 & 50.90 & 59.54 \\
$\varphi=0.04$ & 62.06 & 52.98 & 2.61 & 53.03 & 62.19 \\
\hline
\end{tabular}

fusion convection of nanofluid is reported for the first time, too. We find that a new interesting phenomenon in nanofluid which is obviously different from the pure base fluid. Finally, an original power-like correlation on the heat and mass transfer in double diffusion of nanofluid is extracted through our comprehensive numerical experiments. The resutls presented in this study can answer some critical fundamental questions in double diffusive natural convection in 
Table 10

The computed average Sherwood number at the hot wall when $R a=10^{9}$.

\begin{tabular}{cccccc}
\hline & $N=0$ & $N=0.5$ & $N=1.0$ & $N=1.5$ & $N=2.0$ \\
\hline$\varphi=0$ & 52.97 & 45.47 & 2.12 & 45.44 & 52.99 \\
$\varphi=0.01$ & 52.52 & 44.99 & 2.12 & 45.12 & 52.52 \\
$\varphi=0.02$ & 51.79 & 44.45 & 2.12 & 44.42 & 51.77 \\
$\varphi=0.03$ & 51.04 & 43.69 & 2.12 & 43.67 & 51.08 \\
$\varphi=0.04$ & 50.44 & 43.06 & 2.12 & 43.10 & 50.55 \\
\hline
\end{tabular}

Table 11

The computed average Sherwood number at the hot wall when $R a=10^{10}$.

\begin{tabular}{cccccc}
\hline & $N=0$ & $N=0.5$ & $N=1.0$ & $N=1.5$ & $N=2.0$ \\
\hline$\varphi=0$ & 68.96 & 65.94 & 2.12 & 65.94 & 68.91 \\
$\varphi=0.01$ & 71.03 & 67.85 & 2.19 & 67.87 & 71.03 \\
$\varphi=0.02$ & 74.81 & 71.30 & 2.31 & 71.36 & 74.80 \\
$\varphi=0.03$ & 79.69 & 75.65 & 2.47 & 75.65 & 79.66 \\
$\varphi=0.04$ & 83.81 & 79.42 & 2.61 & 79.41 & 83.76 \\
\hline
\end{tabular}

Table 12

The computed average Sherwood number at the hot wall when $R a=10^{10}$.

\begin{tabular}{cccccc}
\hline & $N=0$ & $N=0.5$ & $N=1.0$ & $N=1.5$ & $N=2.0$ \\
\hline$\varphi=0$ & 68.96 & 65.94 & 2.12 & 65.94 & 68.91 \\
$\varphi=0.01$ & 68.81 & 65.73 & 2.12 & 65.75 & 68.81 \\
$\varphi=0.02$ & 68.59 & 65.38 & 2.12 & 65.43 & 68.60 \\
$\varphi=0.03$ & 68.37 & 64.90 & 2.12 & 64.90 & 68.34 \\
$\varphi=0.04$ & 68.12 & 64.55 & 2.12 & 64.54 & 68.08
\end{tabular}

Table 13

Coefficients in Eq.(30).

\begin{tabular}{lccccc}
\hline & $a$ & $b$ & $c$ & $d$ & $e$ \\
\hline$R a=10^{5}$ & 0.14 & 5.1 & 0.266 & 4.4 & 1.2 \\
$R a=10^{6}$ & 0.18 & 5.1 & 0.266 & 4.4 & 1.2 \\
$R a=10^{7}$ & 0.2 & 5.1 & 0.266 & 4.4 & 1.2 \\
$R a=10^{8}$ & 0.201 & 4.5 & 0.266 & 16.58 & 2.25 \\
$R a=10^{9}$ & 0.205 & 4.5 & 0.266 & 12.6 & 2.1 \\
$R a=10^{10}$ & 0.215 & 5.5 & 0.251 & 12.6 & 2.1 \\
\hline
\end{tabular}

detail and therefore accelerate the industrial application of nanofluid in the relevant fields, such as solar energy. 
Table 14

Coefficients in Eq.(31).

\begin{tabular}{lcccc}
\hline & $\mathfrak{a}$ & $\mathfrak{b}$ & $\mathfrak{c}$ & $\mathfrak{d}$ \\
\hline$R a=10^{5}$ & 0.15 & -3.0 & 0.266 & 1.21 \\
$R a=10^{6}$ & 0.19 & -1.8 & 0.266 & 1.21 \\
$R a=10^{7}$ & 0.21 & -2.2 & 0.266 & 1.21 \\
$R a=10^{8}$ & 0.21 & -2.5 & 0.266 & 2.36 \\
$R a=10^{9}$ & 0.211 & -1.9 & 0.266 & 2.1 \\
$R a=10^{10}$ & 0.218 & -0.1 & 0.251 & 2.12 \\
\hline
\end{tabular}

\section{ACKNOWLEDGE}

This work was supported by the National Natural Science Foundation of China (Grant No. 51176061), the Foundation of State Key Laboratory of Coal Combustion, Fundamental Research Funds for the Central Universities (Grant No. 2014QN189) and the British Newton Alumni Fellowship Scheme.

\section{References}

[1] S. Ozerinc, S. Kakac, A.G. Yazicioglu. Enhanced thermal conductivity of nanofluids: a state-of-the-art review. Microfluid Nanofluid 8 (2010)145-170.

[2] R. parizadlaein, J.A. Esfahani, S.P. Laein. Comparison of Theoretical Prediction and Experimental Values of Nanofluids Thermal Conductivity filled by TiO2, $\mathrm{SiO} 2$, and Al2O3, Philippine Scientist 49 (2013) 32-37

[3] D. Wen,G. Lin, S. Vafaei, K. Zhang. Review of nanofluids for heat transfer applications. Particuology 7 (2009) 141-150.

[4] R. Saidur, K.Y. Leong, H.A. Mohammad. A review on applications and challenges of nanofluids. Renew. Sust. Energ. Rev. 15 (2011) 1646-1668.

[5] Z. Haddad, H. F. Oztop, E. Abu-Nada, A. Mataoui. A review on natural convective heat transfer of nanofluids. Renew. Sust. Energ. Rev. 16 (2012) 53635378 .

[6] S. Rashidi, M. Bovand, J.A. Esfahani. Enhancement of heat transfer by nanofluids and orientations of the equilateral triangular obstacle. Energy Conversion and Management 97 (2015) 212-223

[7] O. Mahian, A. Kianifar, S.A. Kalogirou, I. Pop, S. Wongwises. A review of the applications of nanofluids in solar energy. Int. J. Heat Mass Transfer 57 (2013) 582-594. 
[8] J.A. Esfahani, V. Bordbar. Double diffusive natural convection heat transfer enhancement in a square enclosure using nanofluids. J. Nanotechnology Eng. Med. 2(2011) 021002/1-021002/9.

[9] S. Parvin, R. Nasrin, M.A. Alim, N.F. Hossain. Double diffusive natural convection in a partially heated enclosure using nanofluid. Heat Transf. Asian Res. 41 (2012) 484-497.

[10] M.A. Alim, R. Nasrin. Modeling of Double Diffusive Buoyant Flow in a Solar Collector with Water-CuO Nanofluid. Heat Transf. Asian Res. 42 (2013) 212229 .

[11] D.A. Nield, A.V. Kuznetsov. The onset of double-diffusive convection in a nanofluid layer. Int. J. Heat Fluid Flow 32 (2011) 771-776.

[12] A.V. Kuznetsov, D.A. Nield. Double-diffusive natural convective boundarylayer flow of a nanofluid past a vertical plate. Int. J. Therm. Sci. 50 (2011) 712-717.

[13] D.A. Nield, A.V. Kuznetsov. The Cheng-Minkowycz problem for the doublediffusive natural convective boundary layer flow in a porous medium saturated by a nanofluid. Int. J. Heat Mass Transfer 54 (2011) 374-378.

[14] O.A. Beg, D. Tripathi. Mathematica simulation of peristaltic pumping with double-diffusive convection in nanofluids: a bio-nanoengineering model. J. Nanoengineering Nanosystems DOI: 10.1177/1740349912437087

[15] S. Chen, H. Liu, C.G. Zheng. Numerical study of turbulent double-diffusive natural convection in a square cavity by LES-based lattice Boltzmann model. Int. J. Heat Mass Transf. 55 (2012) 4862-4870.

[16] A. Kamyar, R. Saidur, M. Hasanuzzaman. Application of Computational Fluid Dynamics (CFD) for nanofluids. Int. J. Heat Mass Transfer 55 (2012) 4104-4115

[17] C.J. Ho, M.W. Chen, Z.W. Li. Numerical simulation of natural convection of nanofluid in a square enclosure: Effects due to uncertainties of viscosity and thermal conductivity. Int. J. Heat Mass Transfer 51 (2008) 4506-4516

[18] M. Jahanshahi, S.F. Hosseinizadeh, M. Alipanah, A. Dehghani, G.R. Vakilinejad. Numerical simulation of free convection based on experimental measured conductivity in a square cavity using Water $/ \mathrm{SiO} 2$ nanofluid. Int. Commun. Heat Mass Transfer 37 (2010) 687-694.

[19] C. Beghein, F. Haghighat, F. Allard, Numerical study of double-diffusive natural convection in a square cavity. Int. J. Heat Mass Transfer 35(1992)833846.

[20] L.A. Tofaneli, M.J.S. de Lemos, Double-diffusive turbulent natural convection in a porous square cavity with opposing temperature and concentration gradients. Int. Commun. Heat Mass Transfer 36 (2009) 991-995. 